\title{
Comparative Study between CT and MRI in Assessment of Laryngeal Cartilage Invasion in Laryngeal Carcinoma
}

\author{
SHAIMA F. ELKHOLY, M.D.*; MOHAMED A. KANDEEL, M.Sc.*; \\ MO'MEN A. AMEEN HAMELA, M.D.** and RAMY E. IBRAHIM ASAAD, M.D.* \\ The Departments of Radiology* and ENT**, Faculty of Medicine, Cairo University, Egypt
}

\begin{abstract}
Background: Laryngeal cancer comprises 2 to $5 \%$ of all malignant diseases diagnosed annually worldwide. Cartilage invasion by the laryngeal cancer is of great importance through the staging of laryngeal carcinoma according to TNM classification and has a great impact on management plan. There is a scarcity in the published literature regarding the comparison between MRI and CT for assessment of cartilage involvement.

Aim of Study: To assess the validity of MRI and to compare this with CT findings in predicting inner and outer thyroid cartilage laminae invasion in patients with laryngeal carcinoma.

Patients and Methods: The present study was an observational analytical study that was carried out from January 2018 to June 2019 at the Radiology Department of Faculty of Medicine, Cairo University. A total of 30 patients with laryngeal carcinoma were included. CT and MRI was done for all patients.
\end{abstract}

Results: There was a good agreement between readers in $\mathrm{CT}$ interpretation in term of inner lamina involvement $(\mathrm{K}=0.78)$ and very good agreement in term of outer lamina involvement $(\mathrm{K}=0.87)$. While in MRI interpretation, there was a very good agreement between readers in term of inner lamina involvement $(\mathrm{K}=0.83)$ and outer lamina involvement $(\mathrm{K}=0.92)$. However higher accuracy \& confidence levels were found on interpretation of both CT and MRI together. We found a higher interobserver agreement between interpretation of CT and MRI together $(\mathrm{K}=1.00)$.

Conclusion: MRI showed high validity and precision in detecting inner and outer thyroid lamina invasion by laryngeal caner than CT scan. We found that the combination of CT and MRI together was associated with more accuracy and confidence level for the readers.

Key Words: Cartilage invasion - Laryngeal carcinoma - TNM staging - MRI - CT.

\section{Introduction}

LARYNGEAL carcinoma comprises about 2 to $5 \%$ of the malignant diseases diagnosed annually

Correspondence to: Dr. Shaima F. Elkholy, E-Mail: shaimafatooh@yahoo.com worldwide. Predisposing factors for cancer larynx include tobacco smoking, excessive alcohol consumption, reflux, human papilloma viruses infections and many others factors. The commonest pathological type of cancer larynx is squamous cell carcinoma which is accounting for about 95$96 \%$ of the malignant laryngeal tumors [1].

Invasion of the laryngeal cartilage by the malignant lesion is of great importance in the TNM classification of laryngeal carcinoma, as invasion of the inner cortex of the thyroid cartilage is considered as a T3 tumor, whereas tumor invasion through the outer cortex of the thyroid or cricoid cartilages is a T4 tumor [1].

Such crucial findings have an impact on therapeutic planning. Laryngeal preservation approach is an appropriate surgical treatment option, yet major thyroid cartilage invasion and extra laryngeal spread are generally considered contraindications for laryngeal conservative surgery, whereas minor cartilage invasion is not a contraindication. Invasion of the outer cortex of the thyroid cartilage may be considered as a relative contraindication for radiation therapy or non-surgical organ preservation approaches, whereas minor inner cortex erosion may be amenable to radiation therapy [2] .

Hence, an accurate assessment for potential cartilage invasion by the laryngeal cancer is essen-

\footnotetext{
List of Abbreviations:

DL : Direct Laryngoscopy.

FOV : Field of View.

IRB : Institutional Review Board.

NPV : Negative Predictive Value.

PPV : Positive Predictive Value.

SCC : Squamous Cell Carcinoma.

TE : Time of Echo.

TR : Time of Repetition.

TSE : Turbo Spin-Echo.
} 
tial in daily practice. The sub-mucosal extension of the tumor cannot be detected by endoscopy and also as a result; the accurate tumor size can be underestimated. Some rare tumor entities like chondrosarcoma are typically located in the submucosal space and the assessment of the extensions of such tumor is difficult [3].

Cross-sectional imaging play an important role of staging of the laryngeal cancer. The aim of cross sectional imaging is to detect the deep tumoural extension into laryngeal spaces including the pre epiglottic space, paraglottic space and the thyroarytenoid muscle as well as to detect invasion of the laryngeal cartilages. Of particular importance is the assessment of the suitability for partial laryngectomy and/or chemoradiation therapy in an attempt to preserve the patient's voice by laryngeal conservative surgery [4]. The aim of our work was to assess the validity of MRI compared to CT in detection of the thyroid cartilage invasion in patients with laryngeal carcinoma.

\section{Patients and Methods}

Eligible patients were informed about the study objectives, methodology, risks and benefits. A written informed consent was obtained from every participants prior to the study. The study's protocol was reviewed and approved by IRB, Ethics Committee and audit department of our institution.

The study was a prospective observational study done from January 2018 to June 2019 at the Radiology Department of our Institution. The study is a double blind study where three radiologists interpreted the CT and MRI scans separately to give their opinion then reviewed the cases concomitantly.

The present study included 30 patients who met the study criteria. Each eligible patient underwent full history taking and clinical examination including age, sex, general condition at presentation and cause of admission. Laboratory investigations which include Complete Blood Count (CBC) profile, coagulation profile, liver and renal function tests. All patients underwent both CT and MRI examinations.

Inclusion criteria: Patients who had a clinicopathological diagnosis of laryngeal Squamous Cell Carcinoma (SCC) confirmed through Direct Laryngoscopy (DL). Direct laryngoscopy and histopathological data were obtained to confirm.

Exclusion criteria: A histological diagnosis other than squamous cell carcinoma. History of chemotherapy, radiotherapy or previous surgical intervention for the laryngeal lesion. Contraindication to contrast media and absolute MRI contraindications.

A- CT Protocol: CT scans were obtained using a 64-multislice CT scanner (GE series). Ultravest was used as the contrast medium $(1 \mathrm{ml} / \mathrm{kg}$ body weight). Axial $(0.5-1 \mathrm{~mm})$ cuts were obtained followed by sagittal (3-5mm) and coronal (3$5 \mathrm{~mm}$ ) reconstructed images with both soft tissue and bone windows.

B- MRI Protocol: All MR imaging studies were obtained at Philips 1.5 Tesla MRI machine (Achieva). Included turbo spin-echo images (TSE) sequence in T1 weighted image in axial, coronal and sagittal localizer image plane (TR/ TE 450/11), T2 weighted in axial, coronal and sagittal planes (TR/TE 4200/72) and T2 weighted FS in axial plane (fat saturation, TR/TE 4980/72) and axial proton-density fast spin echo (TR/TE 1,500/38). No contrast media was given. The number of sections for all sequences was 25 , the section thickness was 3-4 mm, intersection gap was $1 \mathrm{~mm}$, and Field of View (FOV) was $240 \mathrm{~mm}$ with acquisition matrix 256-256.

\section{Image interpretation:}

The CT and MRI images were analyzed independently by three radiologist with 12,15 and 23 years experience in head and neck in different situations including the assessment of CT only, then the MRI only and finally both CT and MRI together and they were requested to express their degree of confidence on a five-point scale (ranging from $1=$ No confidence to $5=$ Total confidence). And then the data were collected and analyzed.

$C T$ : The site and extension of lesion, signs of impaired vocal cord mobility and deep laryngeal invasion (para-glottic or pre-epiglottic spaces and thyroid cartilage) were assessed. On CT images, the invasion of thyroid cartilage was defined based on the destruction of the cartilage or presence of just cortical erosions.

MRI: The criteria used to diagnose thyroid cartilage invasion included one or more of the following: Fat obliteration of para-glottic or preglottic spaces, altered signal of the thyroid cartilage seen as isointense to the tumour signal in $\mathrm{T} 1$ and T2 WI or direct invasion or erosion of the thyroid cartilage by the soft tissue mass.

\section{Statistical analysis:}

An Excel spreadsheet was established for the entry of data. We used validation checks on numerical variables and option-based data entry method 
for categorical variables to reduce potential errors. The analyses were carried with SPSS software (Statistical Package for the Social Sciences, version 24, SSPS Inc, Chicago, IL, USA). Frequency tables with percentages were used for categorical variables and descriptive statistics (mean and standard deviation) were used for numerical variables. Either paired Student or Mann-Whitney tests were used to compare quantitative variables, while McNemar or McNemar-Bowker tests were used to analyze categorical variables.

The agreement between readers was examined by calculation of the prevalence and bias-adjusted kappa. As a rule of thumb value of Kappa from 0.40 to 0.59 were considered moderate, 0.60 to 0.79 were considered substantial, and 0.80 were considered outstanding.

\section{Results}

In the present prospective study, we included 30 patients all were males. The mean age of the patients was $61.87 \pm 5.6$ years. Almost $47 \%$ of the patients aged between 50-60 years old, $47 \%$ between 60-70 years old, and 6\% aged more than 70 years old. In addition, all of the included patients were smokers, $66.7 \%$ had diabetes mellitus, and $46.7 \%$ had hypertension.

According to reader 1, CT findings by reader 1 for inner lamina involvement showed 11 positive and 19 negative cases. And for outer lamina involvement were 5 positive and 25 negative cases. The MRI findings by reader 1 for inner lamina involvement showed 22 positive and 8 negative cases. And for outer lamina involvement were 9 positive and 21 negative. On reviewing $\mathrm{CT}$ and MRI together by reader 1, the inner lamina involvement showed 19 positive and 11 negative cases, and for outer lamina involvement were 8 positive and 22 negative cases.

As a conclusion for reader 1 , the interpretation of CT alone revealed 8 false negative cases in term of inner lamina involvement and 3 false negative cases in term of outer lamina involvement. Also, we found that interpretation of MRI alone revealed 3 false positive cases in term of inner lamina involvement and 1 false positive case in term of outer lamina involvement.

According to reader 2, CT findings for inner lamina involvement showed 12 positive and 18 negative cases. And for outer lamina involvement were 6 positive and 24 negative cases. In contrast, the MRI findings by reader 2 for inner lamina involvement showed 23 positive and 7 negative cases, and for outer lamina involvement were 10 positive and 20 negative. On reviewing CT and MRI together by reader 2, the inner lamina involvement showed 19 positive and 11 negative cases. And for outer lamina involvement were 8 positive and 22 negative cases.

As a conclusion, for reader 2, the interpretation of CT only revealed 7 false negative cases in term of inner lamina involvement and 2 false negative cases in term of outer lamina involvement. Also, we found that interpretation of MRI only revealed 4 false positive cases in term of inner lamina involvement and 2 false positive cases in term of outer lamina involvement.

According to reader 3, CT findings for inner lamina involvement showed 12 positive and 18 negative cases. And for outer lamina involvement were 5 positive and 25 negative cases. In contrast, the MRI findings by reader 3 for inner lamina involvement showed 23 positive and 7 negative cases, and for outer lamina involvement were 9 positive and 21 negative. On reviewing $\mathrm{CT}$ and MRI together by reader 3, the inner lamina involvement showed 19 positive and 11 negative cases. And for outer lamina involvement were 8 positive and 22 negative cases.

As a conclusion, for reader 3 , the interpretation of CT only revealed 7 false negative cases in term of inner lamina involvement and 3 false negative cases in term of outer lamina involvement. Also, we found that interpretation of MRI only revealed 4 false positive cases in term of inner lamina involvement and 1 false positive cases in term of outer lamina involvement [findings are summarized in (Table 1)].

\section{Inter observer agreement analysis:}

There was good agreement between readers in $\mathrm{CT}$ interpretation in term of inner lamina involvement $(\mathrm{K}=0.78)$ and very good agreement in term of outer lamina involvement $(\mathrm{K}=0.87)$. There is very good agreement between readers in MRI interpretation term of inner lamina involvement $(\mathrm{K}=0.83)$ and outer lamina involvement $(\mathrm{K}=0.92)$. Higher agreement between the readers is seen on interpretation of $\mathrm{CT}$ and MRI together $(\mathrm{K}=1.00)$.

\section{Analysis of the reader confidence levels (Table 2):}

The confidence levels are ordered to range from 1 to 5 , where 1 refer to non-confident and 5 refer to very confident. According to reader 1 , the mean of confidence value for CT appeared to be (3.86) and for MRI to be (4.23) compared to interpretation of both modalities together that shows higher 
confidence value (mean value 4.61). According to reader 2, the mean of confidence value for CT was (3.56) and for MRI was (4.35) compared to interpretation of both modalities together that show higher confidence value (mean value 4.70). Ac- cording to reader 3 , the mean of confidence value for CT appeared to be (3.89) and for MRI to be (4.26) compared to interpretation of both modalities together with higher confidence value (mean value 4.63). Figs. (1-4).

Table (1): Inner and outer lamina invasion as detected by CT, MRI and both CT \& MRI together for each reader.

\begin{tabular}{|c|c|c|c|c|c|c|}
\hline & \multicolumn{2}{|c|}{ Reader 1} & \multicolumn{2}{|c|}{ Reader 2} & \multicolumn{2}{|c|}{ Reader 3} \\
\hline & Inner lamina & Outer lamina & Inner lamina & Outer lamina & Inner lamina & Outer lamina \\
\hline CT & 11 & 5 & 12 & 6 & 12 & 5 \\
\hline MRI & 22 & 9 & 23 & 10 & 23 & 9 \\
\hline CT \& MRI & 19 & 8 & 19 & 8 & 19 & 8 \\
\hline
\end{tabular}

Table (2): Confidence levels of the three readers for CT, MRI and both CT \& MRI together.

\begin{tabular}{llllllllll}
\hline & \multicolumn{3}{c}{ Reader 1 } & \multicolumn{2}{c}{ Reader 2 } & \multicolumn{2}{c}{ Reader 3 } \\
\cline { 2 - 10 } & CT & MRI & CT \& MRI & CT & MRI & CT \& MRI & CT & MRI & CT \& MRI \\
\hline Mean & 3.86 & 4.23 & 4.61 & 3.56 & 4.35 & 4.7 & 3.89 & 4.26 & 4.63 \\
Minimum & 1 & 2 & 4 & 1 & 3 & 4 & 1 & 2 & 4 \\
Maximum & 5 & 5 & 5 & 5 & 5 & 5 & 5 & 5 & 5 \\
Standard deviation & 1.05 & 0.98 & 0.35 & 1.04 & 1.02 & 0.30 & 1.03 & 1.01 & 0.36 \\
\hline
\end{tabular}
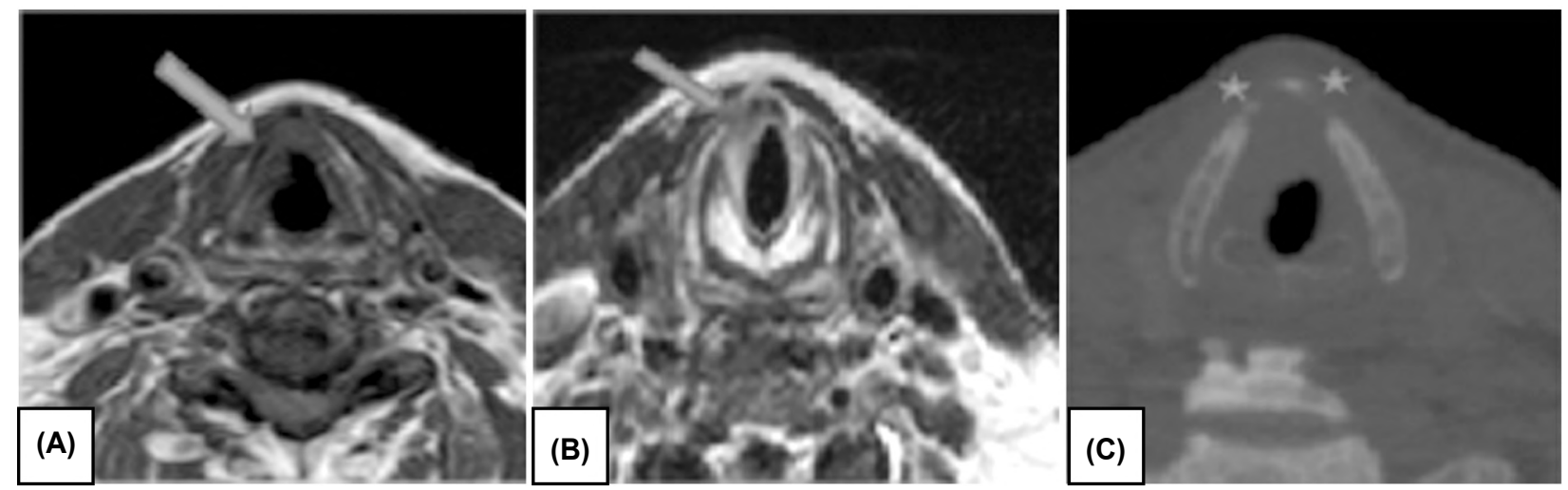

Fig. (1): Male patient 80 years old with pathologically proven laryngeal cancer. (A) Axial T1 and (B) Axial T2 MRI images show anterior thyroid cartilage signal abnormality being isointense to the vocal cord lesion in different sequences more evident on the right side involving both inner and outer lamina (blue arrow) (C) Axial CT image shows right vocal cord lesion with thickened anterior commisure with no extra-laryngeal extension. There's thinning and erosions of the inner and outer lamina of the anterior aspects of both thyroid cartilage suggesting infiltration.
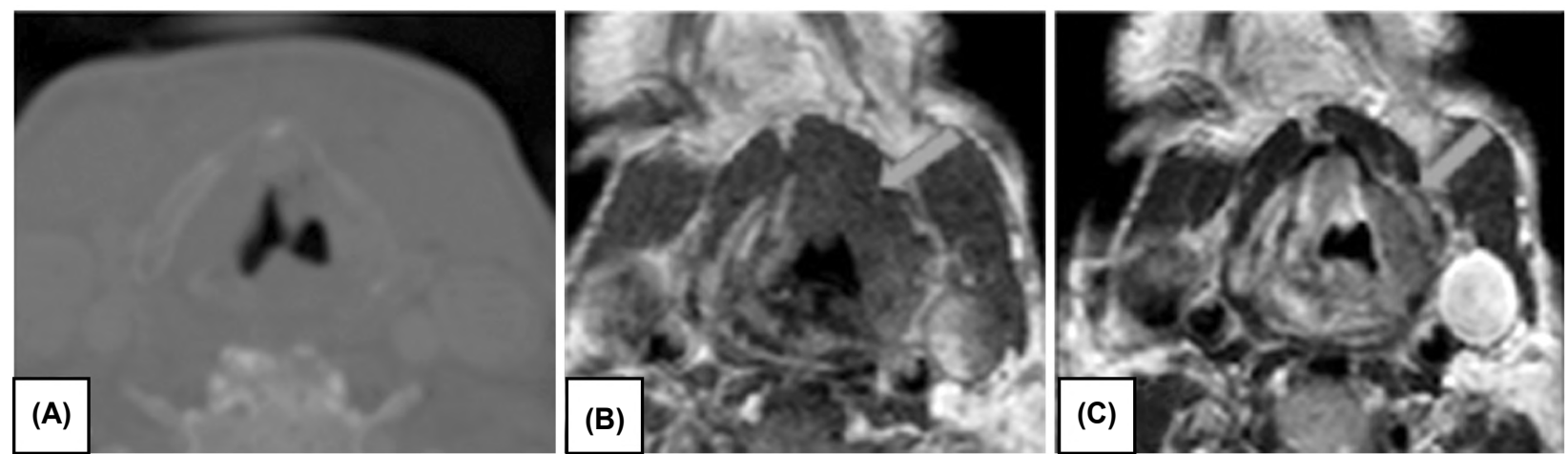

Fig. (2): Male patient 63 years old with pathologically proven laryngeal cancer. (A) Axial CT image shows left vocal cord mass lesion with extension across the anterior commisure. There's thinning and erosions of the inner lamina of the left thyroid cartilage as well as the anterior aspect of the right side suggesting infiltration yet with apparently intact outer lamina. (B) Axial T1 and (C) Axial T2 MRI images show infiltration of both inner and outer lamina more evident on the left side "blue arrow" which was not detected by CT only. 
Fig. (3): Male patient 60 years old with pathologically proven laryngeal cancer. (A) Axial CT image shows right vocal cord mass lesion with obliterated right paraglottic fat planes yet apparently intact thyroid cartilage. (B) Axial T 1 and (C) axial T2 MRI images show abnormal signal intensity of both inner and outer lamina of the right thyroid cartilage denoting its infiltration more along its posterior half "blue arrow" which was not detected by CT only.
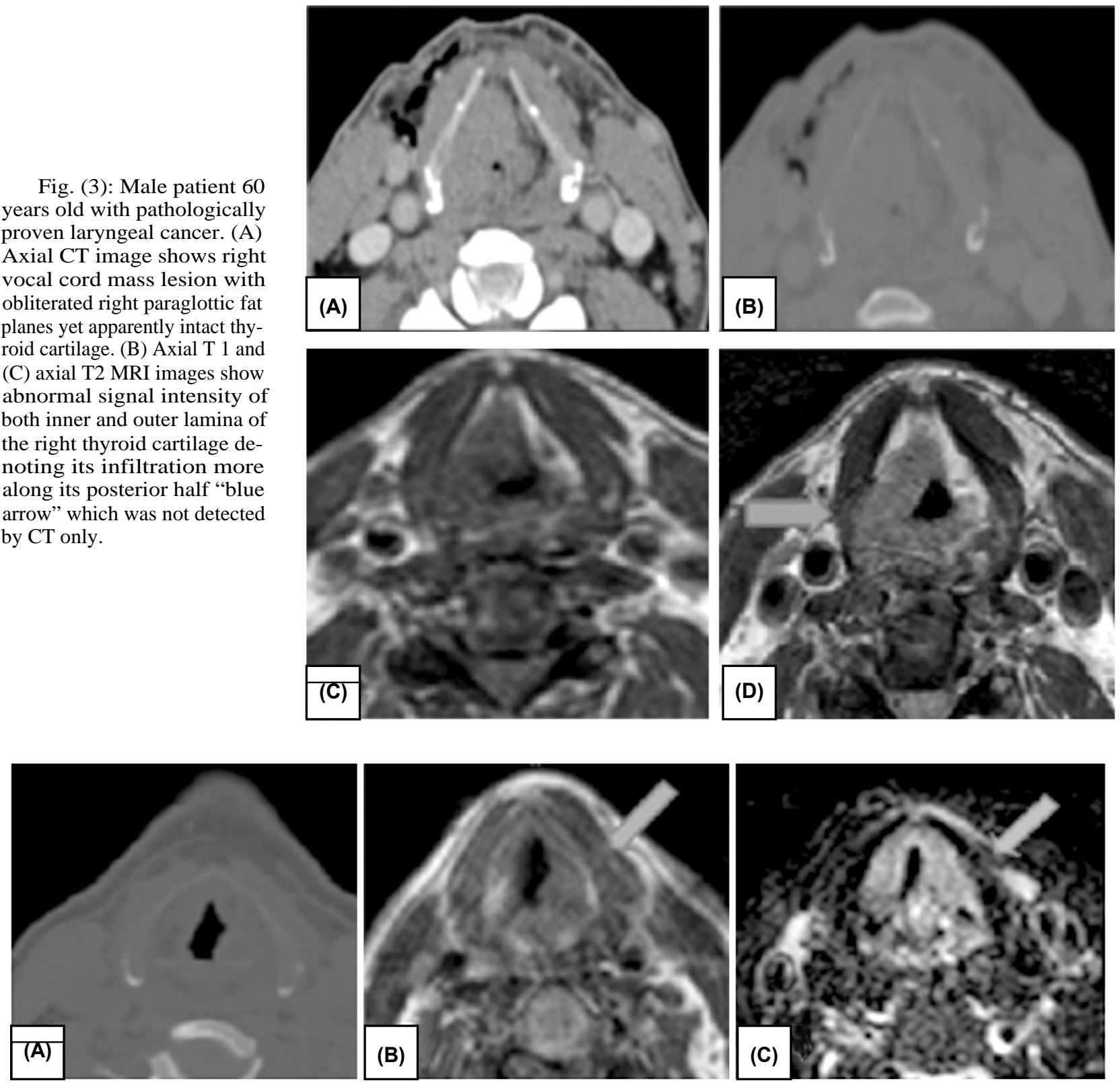

Fig. (4): Male patient 52 years old with pathologically proven laryngeal cancer. (A) Axial CT image shows left vocal cord lesion. The thyroid cartilage is not opacified with no definite invasion. (B) Axial T1 and (C) Axial T2 STIR MRI images show focal infiltration of the inner and outer lamina "blue arrow" more appreciated at STIR images which was not detected by CT only.

\section{Discussion}

Laryngeal cancers is representing about one third of all head and neck cancers and the second most common malignancy of the upper aerodigestive tract. It's considered as one of the significant casues of morbidity and mortality worldiwide [5]

Imprtant criteria for staging of laryngeal carcinoma include vocal cord mobility and tumor extension in addition to laryngeal cartilage invasion. Minor cartilage invasion is considered as T3 tumour while extralaryngeal tumor spread is considered as a T4 tumor. The incidence of thyroid cartilage invasion is reported to be aound $19-27 \%$. Hence, an accurate assessment of potential cartilage invasion is essential in daily practice [6]

Laryngeal preservation surgery indicated for advanced laryngeal tumors needs a correct TNM stage classification. Used in combination with clinical history and laryngoscopy, the accuracy rates of CT and MRI in T staging of laryngeal carcinoma could reach $80 \%$ and $87 \%$ respectively [7]

CT is the commonest cross sectional imaging modality for assessment of local laryngeal cancer 
extensions. Recently, MRI has been introduced as an accurate modality for assessment of the cartilage involvement. MRI has a similar ability to define the interface between fat and tumor, but is superior for assessing muscle and cartilage invasion. In addition, MRI does not require iodinated contrast, has no exposure to ionizing radiation and has superior soft-tissue contrast [8].

Nevertheless, there is a scarcity in the published literature comparing MRI and CT for assessment of cartilage involvement. Therefore, we conducted the present double blinded study in order to assess the validity of MRI in predicting inner and outer thyroid cartilage laminae invasion in patients with laryngeal carcinoma, as compared to CT scan.

All patients included in our study were males. In line with our findings, Abdel Tawab et al., [9] aimed to study the epidemiological aspects of laryngeal cancer on a total of 295 patients and found that $93.9 \%$ (277) of the patients were men.

In the present study, all of the included patients were smokers. Smoking is the most significant risk factor for cancers of the larynx in $70 \%$ to $95 \%$ of all cases. Any history of smoking portends higher risk, with current smokers exhibiting increased relative risk versus ex-smokers overall and increased relative risk for supraglottic versus glottic cancers [10]

In terms of the thyroid lamina involvement in the present study, $19(63.3 \%)$ patients had inner lamina involvement and $8(26.7 \%)$ patients had outer lamina involvement. There was good agreement between readers in term of inner lamina involvement with very good agreement in term of outer lamina involvement on interpretation of the CT and MRI separately with average confidence level and higher values were detected on interpretation of both modalities together.

Li et al., [11] evaluated the values of CT and MRI for the diagnosis of thyroid cartilage invasion by laryngeal and hypopharyngeal cancer. One hundred and ninety-seven patients with primary laryngeal cancer treated with surgery between January 2013 and December 2014 were included in their study. The sensitivity, specificity, positive predictive value and negative predictive value of $\mathrm{CT}$ for the detection of thyroid cartilage invasion were $57 \%, 86 \%, 65 \%$ and $81 \%$ respectively.

Similarly, Dankbaar et al., [12] assessed the diagnostic properties of CT for visual detection of cartilage invasion in laryngeal cancer. Patients with T3 or T4 laryngeal squamous cell carcinoma treated with total laryngectomy were evaluated. The sensitivity, specificity, positive predictive value and negative predictive value of CT for the detection of thyroid cartilage invasion were $67 \%$, $90 \%, 70 \%$ and $89 \%$ respectively.

While other reports showed a much lower sensitivity of CT for prediction of cartilage invasion. For example, Hartl et al., [2] aimed to determine the predictive value of CT for thyroid cartilage invasion in early-to mid-stage laryngeal cancer. Retrospective study (1992-2008) of laryngeal squamous cell carcinoma was conducted and 236 patients were included. CT's sensitivity was $10.5 \%$, specificity $94 \%$, positive predictive value $13 \%$, and negative predictive value $92 \%$. CT correctly predicted thyroid cartilage invasion in only two cases for an overall accuracy of $87 \%$.

Moreover, other reports suggested that CT may lead to over-staging of laryngeal cancer patients by detecting higher rate of cartilage invasion. For example, Li et al., [13] evaluated the Positive Predictive Value (PPV) and Negative Predictive Value (NPV) of detecting neoplastic cartilage invasion in laryngeal cancer patients using fast-speed MultiDetector CT (MDCT). 61 consecutive patients with clinically staged $\mathrm{T} 3$ or $\mathrm{T} 4$ squamous cell carcinoma of the larynx or hypopharynx who underwent total laryngectomy were analyzed. MDCT scan was found to have a PPV of $78 \%$ and an NPV of $100 \%$ for detection of invasion through cartilage, with sensitivity being $100 \%$ and specificity $96 \%$. For detection of any cartilage invasion (minor, major or through cartilage invasion), PPV and NPV were $63 \%$ and $92 \%$, respectively. The sensitivity was $85 \%$ and specificity was $75 \%$. For the detection of tumor invasion through cartilage or major cartilage invasion, MDCT scan had a PPV of 53\% and an NPV of $95 \% .47 \%$ (9/19) patients were down-staged from T4 to T3.

Notably, other studies even reported higher diagnostic accuracy of CT for detection of thyroid cartilage invasion. Dhoot et al., [14] evaluated the adequacy of CT in the assessment of thyroid cartilage invasion in patients with airway cancer. Sixtytwo consecutive patients underwent CT to stage laryngeal $(n=27)$ or hypopharyngeal $(n=35)$ cancer in this prospective study. For thyroid cartilage invasion, CT achieved a sensitivity of $91 \%$ and a specificity of $75 \%$, while US attained a sensitivity of $98 \%$ and a specificity of $75 \%$.

The exact causes of such heterogeneity are unclear. However, this difference can be attributed to different population's characteristics, methodo- 
logical differences and the use of the diagnostic criteria proposed in this study.

In agreement with our findings, Taha et al., [4] assessed the validity of MRI in predicting inner and outer thyroid cartilage laminae invasion in patients with laryngeal carcinoma. A prospective study was carried out between August 2011 and May 2013. The study included 26 patients. The sensitivity, specificity, efficiency, and positive and negative predictive values of MRI for identification of inner thyroid lamina invasion were: $93,82,88$, 88 and $90 \%$ respectively, while those of outer thyroid lamina invasion were: $85,85,85,85$ and $85 \%$, respectively.

Similarly, Li et al., [13] reported that the sensitivity, specificity, positive predictive value and negative predictive value of MRI were respectively $94 \%, 87 \%, 78 \%$ and $97 \%$. Kappa value was 0.77 for MRI in diagnosis of thyroid cartilage invasion.

Moreover, Kuno et al., [15] compared the diagnostic accuracy of MRI and of a combination of weighted images and iodine overlay dual-energy CT images in detecting cartilage invasion by laryngeal and hypopharyngeal squamous cell carcinomas, in particular thyroid cartilage invasion. Fifty-five consecutive patients who underwent 3T MR imaging and 128-slice dual-energy CT for preoperative initial staging of laryngeal or hypopharyngeal squamous cell carcinomas were included. The specificity of MRI for diagnosing thyroid cartilage invasion was $64 \%$ and the sensitivity $100 \%$.

Kinshuck et al., [16] examined the accuracy of MRI in assessing thyroid cartilage and thyroid gland invasion in patients undergoing total laryngectomy for squamous cell carcinoma, by comparing histopathology results with imaging findings. A retrospective study reviewed histology and magnetic resonance scan results for all total laryngectomies performed between 1998-2008 at University Hospital Aintree, Liverpool. Eighty-one magnetic resonance scans were reviewed. There were 22 laryngectomy patients with histologically verified thyroid cartilage invasion and one patient with thyroid gland invasion. There were 31 patients with apparent radiological thyroid cartilage invasion pre-operatively (with 17 false positives), giving sensitivity, specificity, and positive and negative predictive values of $64,71,45$ and $84 \%$, respectively.

Limitation: The relatively small sample size and lack of pathological correlation are the main limitations of the current study. However not all patients underwent surgery and hence we couldn't obtain a definite pathological results to compare it with the findings obtained by CT and MRI.

\section{Conclusion:}

MRI showed high validity and precision in detecting inner and outer thyroid lamina invasion by laryngeal caner than CT scan. We found that the combination of CT and MRI together was associated with more accuracy and confidence level for the readers.

\section{References}

1- ADOLPHS A.P.J., BOERSMA N.A., DIEMEL B.D.M., EDING J.E.C., FLOKSTRA F.E., WEGNER I., et al.: A systematic review of computed tomography detection of cartilage invasion in laryngeal carcinoma. Laryngoscope, 125: 1650-5. doi: 10.1002/lary.25145, 2015.

2- HARTL D.M., LANDRY G., BIDAULT F., HANS S., JULIERON M., MAMELLE G., et al.: CT-scan prediction of thyroid cartilage invasion for early laryngeal squamous cell carcinoma. Eur. Arch. Oto--Rhino-Laryngology, 270: 287-91. doi:10.1007/s00405-012-2005-8, 2013.

3- KOOPMANN M., WEISS D., STEIGER M., ELGES S. RUDACK C. and STENNER M.: Thyroid cartilage invasion in laryngeal and hypopharyngeal squamous cell carcinoma treated with total laryngectomy. Eur. Arch. Oto-Rhino-Laryngology, 273: 3789-94. doi: 10.1007/ s00405-016-4120-4, 2016.

4- TAHA M.S., HASSAN O., AMIR M., TAHA T. and RIAD M.A.: Diffusion-weighted MRI in diagnosing thyroid cartilage invasion in laryngeal carcinoma. Eur. Arch. OtoRhino-Laryngology, 271: 2511-6. doi:10.1007/s00405013-2782-8, 2014.

5- ROSENBLUTH and RICHARD: Geriatric Oncology, An Issue of Clinics in Geriatric Medicine, Vol. 28. No. 1. Elsevier Health Sciences, 2012.

6- WU J.H., ZHAO J., LI Z.H., YANG W.Q., LIU Q.H., YANG Z.Y., et al.: Comparison of CT and MRI in Diagnosis of Laryngeal Carcinoma with Anterior Vocal Commissure Involvement. Sci. Rep., 6. doi:10.1038/srep30353, 2016.

7- JOSHI V., WADHWA V. and MUKHERJI S.: Imaging in laryngeal cancers. Indian J. Radiol. Imaging, 22: 209. doi:10.4103/0971-3026.107183, 2012.

8- HIGAKI T., NAKAMURA Y., TATSUGAMI F., KAICHI Y., AKAGI M., AKIYAMA Y., et al.: Introduction to the technical aspects of computed diffusion-weighted imaging for radiologists. Radiographics, 38: 1131-44. doi: 10.1148/ rg.2018170115, 2018.

9- ABDEL TAWAB H., ABD ELMESSIH M., ALNAGGAR N. and EL SHARKAWY L.: Study of the epidemiology and management of laryngeal cancer in Kasr Al-Aini Hospital. Egypt J. Otolaryngol., 30: 208. doi: 10.4103/ 1012-5574.138468, 2014.

10-KOROULAKIS A. and AGARWAL M.: Laryngeal Cancer. [Updated 2020 Aug. 10]. In: StatPearls [Internet]. Treasure Island (FL): Stat Pearls Publishing; Jan.-. Available from: https://www.ncbi.nlm.nih.gov/books/NBK526076/, 2020 
11- LI H.Y. and X.H. CHEN: "Diagnostic value of enhanced CT/MRI for thyroid cartilage invasion by malignant tumor. Chinese journal of otorhinolaryngology head and neck surgery, 52.5: 372-6, 2017.

12- DANKBAAR J.W., OOSTERBROEK J., JAGER E.A., De JONG H.W., RAAIJMAKERS C.P., WILLEMS S.M., et al.: Detection of cartilage invasion in laryngeal carcinoma with dynamic contrast-enhanced CT. Laryngoscope Investig. Otolaryngol., 2: 373-9. doi:10.1002/lio2.114, 2017.

13-LI B., BOBINSKI M., GANDOUR-EDWARDS R., FARWELL D.G. and CHEN A.M.: Overstaging of cartilage invasion by multidetector CT scan for laryngeal cancer and its potential effect on the use of organ preservation with chemoradiation. Br. J. Radiol., 84: 64-9. doi: 10.1259/ bjr/66700901, 2011.

14- DHOOT N.M., CHOUDHURY B., KATAKI A.C., KA-
KOTI L., AHMED S. and SHARMA J.: Effectiveness of ultrasonography and computed tomography in assessing thyroid cartilage invasion in laryngeal and hypopharyngeal cancers. J. Ultrasound., 20: 205-11. doi: 10.1007/s40477017-0259-0, 2017.

15- KUNO H., SAKAMAKI K., FUJII S., SEKIYA K., OTANI K., HAYASHI R., et al.: Comparison of MR imaging and dual-energy ct for the evaluation of cartilage invasion by laryngeal and hypopharyngeal squamous cell carcinoma. Am. J. Neuroradiol., 39: 524-31. doi: 10.3174/ ajnr.A5530, 2018.

16- KINSHUCK A.J., GOODYEAR P.W.A., LANCASTER J., ROLAND N.J., JACKSON S., HANLON R., et al.: Accuracy of magnetic resonance imaging in diagnosing thyroid cartilage and thyroid gland invasion by squamous cell carcinoma in laryngectomy patients. J. Laryngol. Otol., 126: 302-6. doi: 10.1017/S0022215111003331, 2012.

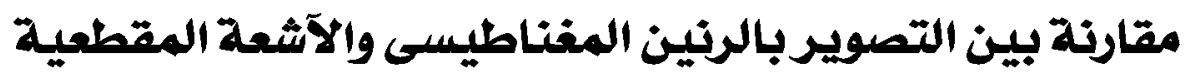

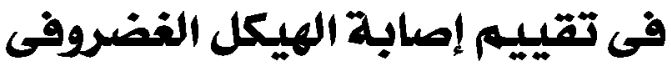

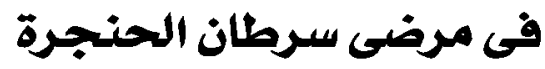

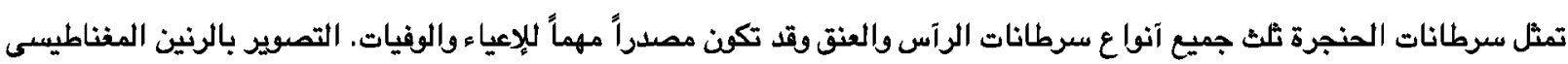

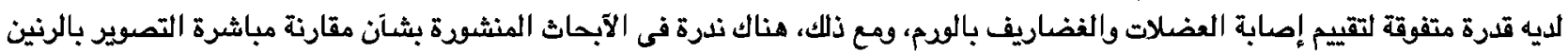

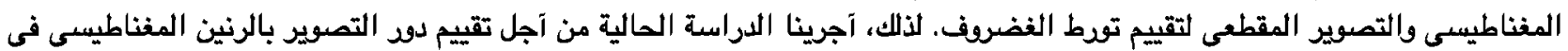
التبؤ بغزو الصفيحة الدرقية الداخلى والخارجى فى المرضى الذين يعانون من سرطان الحنجرة وذلك بالمقارنة مع الآشعة المقطعية.

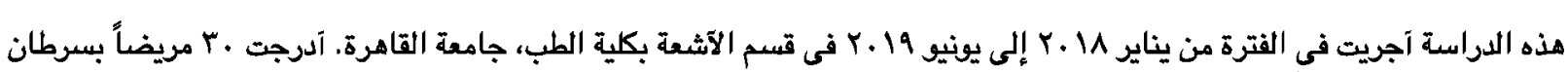
السنجرة تم تصويرهم جميعاً بواسطة الرنئ الرنين المغناطيسى والآشعة المقطعية.

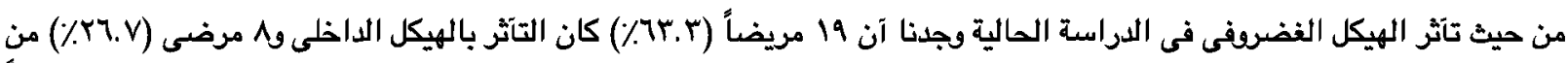

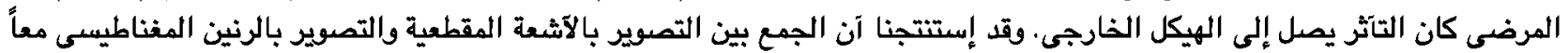

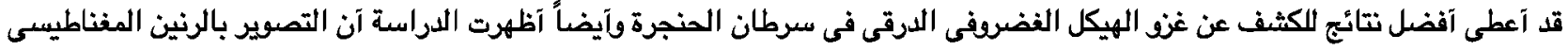

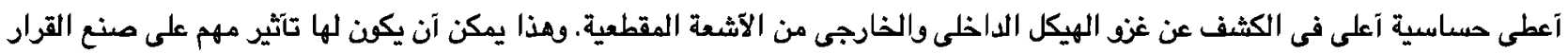

